Session III

PHYSICAL PROCESSES 


\title{
REVIEW OF ATOMIC COLLISION PROCESSES
}

\author{
M.J. SEATON \\ (Dept. of Physics, University College London, England)
}

\section{Recombination Spectra}

\subsection{GENERAL THEORY}

The following processes have to be considered.

(a) Radiative capture,

$$
X^{+}+e \rightarrow X_{i}+h v .
$$

The number of captures on the level $i$ of the atom $X$, per unit volume per unit time, is $N_{\mathrm{e}} N\left(X^{+}\right) \alpha_{i}\left(T_{\mathrm{e}}\right)$, where the recombination coefficient $\alpha_{i}\left(\mathrm{~cm}^{3} \mathrm{sec}^{-1}\right)$ may be expressed in terms of the cross-section for photo-ionization from level $i$.

(b) Cascade,

$$
X_{i^{\prime}} \rightarrow X_{i}+h v_{i^{\prime} i} .
$$

The coefficients $\alpha_{i}^{(\mathrm{c})}$ and $\alpha_{i^{\prime} i}$ are defined to be such that $N_{\mathrm{e}} N\left(X^{+}\right) \alpha_{i}^{(\mathrm{c})}$ is the number of atoms entering $i$ due to capture and cascade, and that $N_{\mathrm{e}} N\left(X^{+}\right) \alpha_{i^{\prime} i}$ is the number of photons emitted in the $i^{\prime} \rightarrow i$ line.

(c) Collisional transitions between excited states, including: collisional redistribution of angular momentum; collisional redistribution of energy between highly excited states; and collision processes which depopulate metastable states.

(d) Effects of radiative transfer. The following limiting cases have been considered:

Case A: Nebulae optically thin in all spectrum lines.

Case B: Nebulae optically thick for transitions to the ground state.

Case C: As Case B, but with absorption of stellar quanta in transitions from the ground state, assuming the central star to radiate as a black body.

\subsection{HYDROGENIC SYSTEMS}

Transition probabilities (Baker and Menzel, 1938; Green et al., 1957) and recombination coefficients (Burgess, 1964) are known exactly for hydrogenic systems.

\subsubsection{Collisional Redistribution of Angular Momentum}

In all earlier calculations (Baker and Menzel, 1938) it was assumed implicitly that angular momentum is completely redistributed before radiation is emitted. With this

Osterbrock and O'Dell (eds.), Planetary Nebulae, 129-137. (c) I.A.U. 
assumption, the most accurate calculations are those of Seaton (1959). Calculations assuming no redistribution of angular momentum have been made by Searle (1958) for $n \leqslant 10$, by Burgess (1958) for $n \leqslant 12$, and by Pengelly (1964a) for an infinite number of levels. These calculations give stronger intensities for transitions of the type $n \rightarrow n-1$. Pengelly and Seaton (1964) have shown that collisional redistribution of angular momentum, due to processes such as

$$
X_{n_{l}}+\mathrm{H}^{+} \rightarrow X_{n_{l \pm 1}}+\mathrm{H}^{+},
$$

is important for the higher states. With $N_{\mathrm{e}}=10^{4} \mathrm{~cm}^{-3}$ collisional redistribution occurs before emission of radiation for $n \gtrsim 15$ in $\mathrm{H}$ and for $n \gtrsim 22$ in $\mathrm{He}^{+}$. Table 1 gives effec-

\section{Table 1}

\section{Effective recombination coefficients for hydrogenic ions}

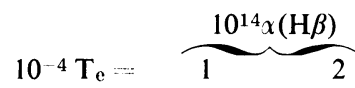

(a) High density $\quad 3.00 \quad 1.60$

(b) Low density $\quad 3.07 \quad 1.64$

(c) $\mathrm{N}_{\mathrm{e}}=10^{4} \mathrm{~cm}^{-3} \quad 3.02 \quad 1.60$

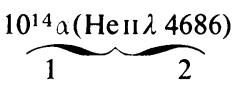

$\begin{array}{ll}20 \cdot 8 & 11 \cdot 8 \\ 37 \cdot 8 & 18.0 \\ 29.4 & 14.8\end{array}$

tive recombination coefficients for $\mathrm{H} \beta$ and for He II $\lambda 4686(n=4 \rightarrow n=3)$, assuming

(a) complete redistribution of angular momentum (limit of high density),

(b) no redistribution of angular momentum (limit of low density),

(c) taking explicit account of redistribution of angular momentum for $N_{\mathrm{e}}=10^{4}$ $\mathrm{cm}^{-3}$ (Pengelly, 1964b).

It is seen that the $\mathrm{H} \beta$ line is not sensitive to the assumptions made, but that the HeII line is much more sensitive.

The calculations of Burgess (1958) allow for complete redistribution of angular momentum for $n \leqslant 12$, and no redistribution for $n>12$. They are the best results to use for $\mathrm{HI}$ at densities of order $10^{4} \mathrm{~cm}^{-3}$.

\subsubsection{Collisional Redistribution of Energy}

Allowance for collisional redistribution of energy must be made in calculations of the populations of highly excited states. Approximate calculations (Seaton, 1964) for $N_{\mathrm{e}}=10^{4} \mathrm{~cm}^{-3}$ indicate that such processes are important only for $n \gtrsim 50$ and may therefore be neglected in considering observed intensities of resolved hydrogen lines in the visible spectrum. A check on the theory should be possible from observations of the intensity due to blended lines within a few Ångstroms of the Balmer limit. Populations of highly excited states are also of interest in connection with the lines observed at radio wavelengths (Goldberg, 1966). Further calculations have been made by Hayler (1967) and by McCarroll and Hoang Binh-Dy (1968). 


\subsection{NON-HYDROGENIC SYSTEMS}

In calculating recombination spectra one is concerned with atomic systems having a single valence electron in excited states. Transition probabilities can be calculated using the method of Bates and Damgaard (1949) and photo-ionization cross-sections can be obtained using the method of Burgess and Seaton (1960a). Improved tabulations of the quantities required for the photo-ionization calculations are given by Peach (1967).

\subsubsection{Helium}

Complications arise from the metastability of $2{ }^{1} \mathrm{~S}$ and $2{ }^{3} \mathrm{~S}$.

(a) Determination of helium abundances: Effects arising from the metastability of $2^{1} \mathrm{~S}$ and $2^{3} \mathrm{~S}$ have little influence on the lines due to transitions $n d \rightarrow 2 p$, and these lines should therefore be used for determinations of helium abundances. Table 2 gives

Table 2

\section{Effective recombination coefficients for HeI lines}

\begin{tabular}{rcl}
$10^{-4} \mathrm{~T}_{\mathrm{e}}=$ & \multicolumn{1}{c}{1} & \multicolumn{1}{c}{2} \\
$10^{14} \alpha\left(\mathrm{He}_{\mathrm{I}} \lambda 5876\right)$ & $5 \cdot 21$ & $2 \cdot 30$ \\
$10^{14} \alpha\left(\mathrm{HeI}_{\mathrm{I}} \lambda 447\right)$ & 1.35 & 0.644
\end{tabular}

recombination coefficients for $3{ }^{3} \mathrm{D} \rightarrow 2{ }^{3} \mathrm{P} \lambda 5876$ and $4{ }^{3} \mathrm{D} \rightarrow 2{ }^{3} \mathrm{P} \lambda 4471$, calculated by Pengelly (1964b) using accurate transition probabilities and photo-ionization crosssections, and neglecting collisional redistribution of angular momentum. The calculated intensity ratio $I(5876) / I(4471)$ is 2.9 at $1 \times 10^{4}{ }^{\circ} \mathrm{K}$ and 2.7 at $2 \times 10^{4}{ }^{\circ} \mathrm{K}$, compared with a mean value of $2 \cdot 5$ for 7 nebulae observed by O'Dell (1963), corrected for reddening. Neglect of collisional redistribution will lead to $\alpha\left(3^{3} \mathrm{D} \rightarrow 2{ }^{3} \mathrm{P}\right)$ being slightly overestimated but will have little effect on $\alpha\left(4{ }^{3} \mathrm{D} \rightarrow 2{ }^{3} \mathrm{P}\right)$.

(b) Self-absorption: Effects due to absorption of radiation in helium lines ending on $2{ }^{3} \mathrm{~S}$ have been considered by Pottasch (1961), Osterbrock (1964), and Capriotti (1967). When the optical depth is suitably chosen there is good agreement with observations for the intensities of $3{ }^{3} \mathrm{P} \rightarrow 2{ }^{3} \mathrm{~S} \lambda 3889$ and $3{ }^{3} \mathrm{~S} \rightarrow 2{ }^{3} \mathrm{P} \lambda 7065$, relative to $\lambda 5876$, but self-absorption effects do not explain the very large intensities observed for $2{ }^{3} \mathrm{P} \rightarrow 2{ }^{3} \mathrm{~S}$ $\lambda 10830$.

(c) Collisional effects: In order to calculate the population of $2{ }^{3} \mathrm{~S}$, and to explain the intensity of $\lambda 10830$, the following processes must be considered: capture and cascade; collisional transitions between all $n=2$ states; depopulation of $2{ }^{3} \mathrm{~S}$ by twophoton emission and by photo-ionization. Accurate cross-sections for transitions between the $n=2$ states, produced by electron impact, have been calculated by Burke et al. (1967a) and these have been used to obtain the rate coefficients $q\left(\mathrm{~cm}^{3} \mathrm{sec}^{-1}\right)$ given in Table 3. In this table we include values of the recombination coefficients 
Table 3

Reaction rates required for the calculation of $\mathrm{HeI} \lambda 10830$ intensities

\begin{tabular}{|c|c|c|c|c|}
\hline & $10^{-4} \mathrm{~T}_{\mathrm{e}}=$ & 1.0 & 1.5 & $2 \cdot 0$ \\
\hline $10^{8} q\left(2^{3} S \rightarrow 2^{1} S\right)$ & & $3 \cdot 1$ & $3 \cdot 5$ & $3 \cdot 4$ \\
\hline $10^{8} q\left(2^{3} \mathrm{~S} \rightarrow 2^{1} \mathrm{P}\right)$ & & 1.4 & $2 \cdot 4$ & $3 \cdot 1$ \\
\hline $1^{8} q\left(2^{3} \mathrm{~S} \rightarrow 2^{3} \mathrm{P}\right)$ & & 20 & 38 & 53 \\
\hline $10^{8} q\left(2^{1} \mathrm{~S} \rightarrow 2^{1} \mathrm{P}\right)$ & & 79 & 122 & 153 \\
\hline $10^{8} q\left(2^{1} \mathrm{~S} \rightarrow 2^{3} \mathrm{P}\right)$ & & $9 \cdot 9$ & $10 \cdot 1$ & $10 \cdot 1$ \\
\hline $10^{14} a^{(c)}\left(2^{3} S\right)$ & & $21 \cdot 0$ & $15 \cdot 0$ & $12 \cdot 0$ \\
\hline $10^{14} \alpha^{(\mathrm{c})}\left(2^{3} \mathrm{P}\right)$ & & $14 \cdot 4$ & $10 \cdot 0$ & $7 \cdot 8$ \\
\hline$I(10830)\left\{N_{e} \rightarrow 0\right.$ & & 1.50 & 1.66 & 1.8 \\
\hline $\mathrm{I}(5876) \quad \mathrm{N}_{\mathrm{e}} \rightarrow \infty$ & & $11 \cdot 1$ & $17 \cdot 6$ & $24 \cdot 7$ \\
\hline
\end{tabular}

$\alpha^{(c)}\left(2{ }^{3} \mathrm{~S}\right)$ and $\alpha^{(\mathrm{c})}\left(2{ }^{3} \mathrm{P}\right)$, and values of the ratio $I(10830) / I(5876)$ calculated in the limit of low density (collisional excitation neglected) and the limit of high density (two-photon emission and photo-ionization neglected).

\subsubsection{Ions of Oxygen and Carbon}

Calculations of recombination spectra have been made by Burgess and Seaton (1960b) for OIII, OIV and Ov and by Pengelly (1964b) for CII, CiII and Civ.

(a) Oxygen ions: In Table 4 we give relative abundances of ions of hydrogen, helium

\section{Table 4}

\section{Ion abundances in NGC 7027}

$\left.\begin{array}{lc}\text { Ion } & \text { Relative abundance } \\ \mathrm{H}^{+} & 100000 \\ \mathrm{He}^{+} & 10900 \\ \mathrm{He}^{+2} & 5100 \\ \mathrm{O}^{0} & 0 \cdot 4 \\ \mathrm{O}^{+} & 1 \cdot 0 \\ \mathrm{O}^{+2} & 15\end{array}\right\}$ from forbidden lines

and oxygen in NGC 7027. The abundances of $\mathrm{O}^{\circ}, \mathrm{O}^{+}$and $\mathrm{O}^{+2}$ are obtained from forbidden lines, and the abundances of $\mathrm{O}^{+3}, \mathrm{O}^{+4}$ and $\mathrm{O}^{+5}$ are obtained on interpreting the observed permitted lines of OIII, OrV and Ov using recombination theory. Since the ionization potential of $\mathrm{O}^{+2}(54.89 \mathrm{eV})$ is close to the ionization potential of $\mathrm{He}^{+}(54.40 \mathrm{eV})$ we would expect the ion abundances to be such that

$$
\frac{N\left(\mathrm{He}^{+}\right)}{N\left(\mathrm{He}^{+2}\right)}=\frac{N\left(\mathrm{O}^{+}\right)+N\left(\mathrm{O}^{+2}\right)}{N\left(\mathrm{O}^{+3}\right)+N\left(\mathrm{O}^{+4}\right)+N\left(\mathrm{O}^{+5}\right)} .
$$


From Table 4 we obtain $2 \cdot 1$ for the ratio on the left-hand side and $0 \cdot 16$ for the ratio on the right-hand side. The helium-ion abundances, and the abundances from forbidden lines should be reliable, and it therefore appears that the abundances of $\mathrm{O}^{+3}, \mathrm{O}^{+4}$ and $\mathrm{O}^{+5}$ obtained from recombination theory are too large by an order of magnitude.

(b) Carbon ions: A similar result is obtained from the calculations for $\mathrm{C}$ ions. Interpreting observed CII, CIII and CIV intensities in NGC 2392, 7027 and 7662 using recombination theory, Pengelly (1964b) obtains carbon/hydrogen abundance ratios of about $5 \times 10^{-3}$ compared with ratios of about $2 \times 10^{-4}$ for typical stellar atmospheres (Aller, 1961). Unless it is assumed that all of these nebulae are carbon-rich, it must be concluded that the abundances obtained using recombination theory are much too large.

\subsection{CASE C}

Since the original work of Baker et al. (1938), little attention has been paid to Case C. It was pointed out by Aller et al. (1939) that the surface brightness must be low if Case $\mathrm{C}$ has to be considered for the hydrogen lines. We consider Case $\mathrm{C}$ for heavier elements.

Let us define a ratio

$R\left(X^{+m}\right)=\frac{\text { (number of excitations of } X^{+m} \text { by line absorption of stellar quanta). }}{\text { (number of excitations of } X^{+m} \text { by recombination) }}$

For an ion such as $\mathrm{O}^{+2}$ it is shown by Burgess and Seaton $(1960 b)$ that optical depths will be large for transitions ending on the ground state. The number of excitations by line absorption of stellar quanta is then largely determined by the Doppler widths of the lines and is insensitive to the ion abundance. The number of recombinations is proportional to the abundance of $X^{+m+1}$ and to the electron density. It follows that the ratio $R\left(X^{+m}\right)$ for a heavy element will be much larger than the ratio $R\left(\mathrm{H}^{0}\right)$

Table 5

NGC 7027, Ratio, $\mathrm{R}\left(\mathrm{O}^{2+}\right)$, of number of $\mathrm{O}^{2+}$ excitations by line absorption of stellar quanta to number by recombination

$\begin{array}{ccc}\tau(\mathrm{H}) & T_{\mathrm{S}}=1 \times 10^{5} & T_{\mathrm{S}}=2 \times 10^{5} \\ 1 & & \\ 5 & 12 & 48 \\ 10 & 4 & 16 \\ \infty & 3 & 11 \\ & 2 & 5\end{array}$

for hydrogen. Table 5 gives the results of approximate calculations of $R\left(\mathrm{O}^{+2}\right)$ for NGC 7027, as a function of optical depth at the Lyman limit, $\tau(\mathrm{H})$, and of the star temperature, $T_{s}$ (Seaton, 1968). It appears that this ratio may be of order 10 , and hence that excitation by absorption of stellar radiation in spectrum lines is much more 
important than excitation by recombination. A similar result may be expected for other ions, and this would seem to give a satisfactory explanation of the results for oxygen and carbon ion abundances discussed in Section 1.3.

Effects of Case $\mathrm{C}$ will be much less important for hydrogen lines but should be considered if calculations are being compared with observations of high accuracy.

\section{Collisional Excitation}

Collisional excitation is responsible for the production of the forbidden lines in the visible spectrum, and may also be expected to be responsible for excitation of permitted lines in the ultraviolet (Osterbrock, 1963; Hummer and Seaton, 1964; Flower, 1968).

Cross-sections $Q(i \rightarrow j)$ are conveniently expressed in terms of collision strengths $\Omega(i, j)$, first introduced by Hebb and Menzel (1940):

$$
Q(i \rightarrow j)=\frac{\pi}{\omega_{i}}\left(\begin{array}{c}
\hbar \\
m v_{i}
\end{array}\right)^{2} \Omega(i, j),
$$

where $v_{i}$ is the velocity of the incident electron and $\omega_{i}$ is the statistical weight of ion level $i$. The collision strengths are dimensionless and symmetrical in initial and final states. Put $E_{i}=\frac{1}{2} m v_{i}^{2}$ and $E_{j}=\frac{1}{2} m v_{j}^{2}$, where $v_{j}$ is the velocity of the scattered electron. The rate coefficient for de-excitation is

$$
q(j \rightarrow i)=\frac{8.63 \times 10^{-6} \Upsilon(i, j)}{\omega_{j} T_{\mathrm{e}}^{1 / 2}} \mathrm{~cm}^{3} \sec ^{-1} \quad\left(E_{i}<E_{j}\right),
$$

where $T_{\mathrm{e}}$ is in ${ }^{\circ} \mathrm{K}$ and

$$
r(i, j)=\int_{0}^{\infty} \Omega(i, j) \exp \left(-E_{i} / k T_{\mathrm{e}}\right) \mathrm{d}\left(E_{i} / k T_{\mathrm{e}}\right) .
$$

If $\Omega$ is independent of energy, $\Upsilon=\Omega$. The rate coefficient for excitation is

$$
q(i \rightarrow j)={\stackrel{\omega}{\omega_{j}}}_{\omega_{i}} q(j \rightarrow i) \exp \left[-\left(E_{j}-E_{i}\right) / k T_{\mathrm{e}}\right] \quad\left(E_{i}<E_{j}\right)
$$

\subsection{THE FORBIDDEN LINES}

A formulation of the collision problem was given by Seaton in 1953 and calculations were made for $\mathrm{O}^{0}, \mathrm{O}^{+}, \mathrm{O}^{+2}, \mathrm{~N}^{+}, \mathrm{Ne}^{+2}$ and $\mathrm{S}^{+}$. The results obtained, together with approximate estimates for other ions, were summarized by Seaton in 1958 and have been used extensively for the interpretation of forbidden-line intensities. All of this earlier cross-section work is now superseded by the results of more recent calculations. 


\subsubsection{Results for Neutral Atoms}

Smith et al. (1967) have given a formulation of the collision problem which is similar to that of Seaton (1953), and have made calculations for $\mathrm{C}^{0}, \mathrm{~N}^{0}$ and $\mathrm{O}^{0}$ which are much more complete and accurate. Their results, which are given in Table 6 , are not very different from the earlier results.

Table 6

\section{Collision strengths for neutral $\mathbf{C}, \mathbf{N}$ and $\mathbf{O}$}

(Levels 1,2 and 3 are the ground configuration terms: ${ }^{3} \mathrm{P},{ }^{1} \mathrm{D}$ and ${ }^{1} \mathrm{~S}$ for $\mathrm{C}$ and $\mathrm{O}$; ${ }^{4} \mathrm{~S},{ }^{2} \mathrm{D}$ and ${ }^{2} \mathrm{P}$ for $\mathrm{N}$. Values of $\mathrm{E}_{1}$ in Rydbergs.)

$E_{1}$

$\begin{array}{rrrr}.2 & 2.94 & .01 & .05 \\ .3 & 4.69 & .46 & .40 \\ .4 & 5.72 & .73 & .64 \\ .5 & 6.42 & .90 & .84 \\ .6 & 6.89 & 1.00 & .99 \\ .8 & 7.56 & 1.14 & 1.19 \\ 1.0 & 8.03 & 1.23 & 1.29\end{array}$

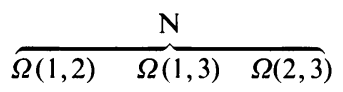

$\begin{array}{rrr}- & - & - \\ 1.01 & - & - \\ 1.78 & .21 & .79 \\ 2.18 & .56 & 1.69 \\ 2.46 & .79 & 2.40 \\ 2.83 & 1.07 & 3.45 \\ 3.08 & 1.24 & 4.13\end{array}$

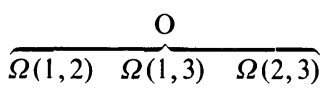

.29

.92

1.40

1.73

1.97

$2 \cdot 28$

2.52

$\begin{array}{rr}- & - \\ - & - \\ .08 & .14 \\ .15 & .21 \\ .21 & .27 \\ .27 & .38 \\ .32 & .45\end{array}$

\subsubsection{Results for Positive Ions}

Results obtained in an extensive programme of new calculations are reported in a separate paper (Czyzak et al., 1968).

Calculations for transitions between fine structure levels, using methods of quantum defect theory, have been made by Osterbrock (1966).

\subsection{THE PERMITTED LINES}

It is to be expected that lines due to resonance transitions in Li-like ions and Na-like ions will be strong in the ultraviolet spectra of high excitation planetaries. Accurate collision strengths are available and are quoted by Flower (1968). Approximate estimates of collision strengths for other ultraviolet lines are given by Osterbrock (1963); some of these are very uncertain.

As a consequence of the $l$-degeneracy of the $\mathrm{H}$ atom, cross-sections for electronimpact excitation remain finite at threshold. In the near-threshold region the best estimates of the cross-sections are given by Burke et al. (1968).

A more detailed discussion of the subject matter of this review is being published elsewhere (Seaton, 1968). 


\section{References}

Aller, L.H. (1961) The Abundance of the Elements, Interscience, Publ., New York and London, p. 115

Aller, L.H., Baker, J.G., Menzel, D.H. (1939) ～Astrophys. J., 89, 587.

Baker, J.G., Menzel, D. H. (1938) Astrophys. J., 88, 52.

Baker, J.G., Menzel, D. H., Aller, L.H. (1938) Astrophys. J., 88, 422.

Bates, D. R., Damgaard, A. (1949) Phil. Trans. R. Soc. Lond., A, 242, 101.

Burgess, A. (1958) Mon. Not. R. astr. Soc., 118, 477.

Burgess, A. (1964) Mem. R. astr. Soc., 69, 1.

Burgess, A., Seaton, M.J. (1960a) Mon. Not. R. astr. Soc., 120, 121.

Burgess, A., Seaton, M.J. (1960b) Mon. Not. R. astr. Soc., 121, 471.

Burke, P.G., Cooper, J.W., Ormonde, S., Taylor, A.J. (1967a) 5th Int. Conf. on Physics of Electronic and Atomic Collisions, Abstracts of Papers, Publishing House Nauka, Leningrad, p. 376.

Burke, P.G., Taylor, A.J., Ormonde, S., Whitaker, W. (1967b) ibid., p. 368.

Capriotti, E.R. (1967) Astrophys. J. (in press).

Czyzak, S.J., Krueger, T.K., Martins, P. de A.P., Saraph, H.E., Seaton, M.J., and Shemming, J. (1968) in the present volume, p. 138.

Flower, D.R. (1968) in the present volume, p. 77.

Goldberg, L. (1966) Astrophys. J., 144, 1225.

Green, L.C., Rush, P.P., Chandler, C.D. (1957) Astrophys. J. Suppl. Ser., 3, 37.

Hayler, D. (1967) Astrophys. J., 150, 95.

Hebb, M.H., Menzel, D.H. (1940) Astrophys. J., 92, 408.

Hummer, D.G., Seaton, M.J. (1964) Mon. Not. R. astr. Soc., 127, 217.

McCarroll, R., Hoang Binh Dy (1968) in the present volume, p. 153.

O'Dell, C.R. (1963) Astrophys. J., 138, 1018.

Osterbrock, D.E. (1963) Planet. Space Sci., 11, 621.

Osterbrock, D.E. (1964) Rev. Astr. Astrophys. 2, 95.

Osterbrock, D.E. (1966) Astrophys. J., 142, 1423.

Peach, G. (1967) Mem. R. astr. Soc., 71, 1.

Pengelly, R. M. (1964a) Mon. Not. R. astr. Soc., 127, 145.

Pengelly, R.M. (1964b) Thesis, London University.

Pengelly, R. M., Seaton, M.J. (1964) Mon. Not. R. astr. Soc., 127, 165.

Pottasch, S.R. (1961) Astrophys. J., 135, 385.

Searle, L. (1958) Astrophys. J., 128, 489.

Seaton, M.J. (1953) Phil. Trans. R. Soc. Lond. A 245, 469.

Seaton, M.J. (1958) Rev. mod. Phys., 30, 979.

Seaton, M.J. (1959) Mon. Not. R. astr. Soc., 119, 90.

Seaton, M.J. (1964) Mon. Not. R. astr. Soc., 127, 177.

Seaton, M.J. (1968) in Advances in Atomic and Molecular Physics, Ed. by D. R. Bates and I. Estermann (in press).

Smith, K., Henry, R.J.W., Burke, P.G. (1967) Phys. Rev., 157, 51.

\section{DISCUSSION}

Aller: Hydrogen recombination rates and level populations have been calculated by Clarke. Three cases of different values of $n_{\text {coll }}$ (the value of the principal quantum number, $n$, at which collisional redistribution is important) viz. $n_{\text {coll }}=0,20$, and 60 are considered, for both HeII and HI. Some results are reported in Vol. 7 of Stars and Stellar Systems (in press).

The CII $\lambda 4267$ line served to suggest a reasonable carbon abundance in the Orion Nebula (W. Liller and L.H. Aller, Astrophys. J., 130, 1959, 45) but carbon recombination calculations for lines of $\mathrm{C}_{\mathrm{II}}, \mathrm{C}_{\mathrm{III}}, \mathrm{C}_{\mathrm{IV}}$ in high-excitation planetaries suggested high abundances of C. I thought these results were due to our rather primitive calculations. Clarke has re-examined the problem but his 
results are not yet available. It should be pointed out that in CIv transitions like $3^{2} \mathrm{~S}-3^{2} \mathrm{P}$ and $5^{2} \mathrm{~F}-6^{2} \mathrm{G}$, which arise from very different levels of excitation, are observed. Accurate intensities interpreted by theory might discriminate between the two rival hypotheses of excitation by fluorescence and by recombination.

Intensities of the OII lines interpreted as arising from recombination give the concentration of $\mathrm{O}^{++}$ions which can also be calculated from the intensities of the [O $\left.\mathrm{III}_{1}\right]$ lines. Cox has been looking into this problem, and should be able to obtain a direct check on the mechanism by computing the $\mathrm{O}^{++}$ionic concentration in the two ways. 